\title{
Review
}

\section{Implications of glutathione-S transferase P1 in MAPK signaling as a CRAF chaperone: In memory of Dr. Irving Listowsky}

\author{
By Yoshiro NiITsu, ${ }^{* 1, * 2, \dagger}$ Yasushi SATO ${ }^{* 3}$ and Tetsuji TAKaYAma ${ }^{* 3}$ \\ (Edited by Shigekazu NAGATA, M.J.A.)
}

\begin{abstract}
Glutathione-S transferase P1 (GSTP1) is one of the glutathione-S transferase isozymes that belong to a family of phase II metabolic isozymes. The unique feature of GSTP1 compared with other GST isozymes is its relatively high expression in malignant tissues. Thus, clinically, GSTP1 serves as a tumor marker and as a refractory factor against certain types of anticancer drugs through its primary function as a detoxifying enzyme. Additionally, recent studies have identified a chaperone activity of GSTP1 involved in the regulation the function of various intracellular proteins, including factors of the growth signaling pathway. In this review, we will first describe the function of GSTP1 and then extend the details onto its role in the mitogen-activated protein kinase signal pathway, referring to the results of our recent study that proposed a novel autocrine signal loop formed by the CRAF/GSTP1 complex in mutated KRAS and BRAF cancers. Finally, the possibilities of new therapeutic approaches for these cancers by targeting this complex will be discussed.
\end{abstract}

Keywords: GSTP1, KRAS cancer, BRAF cancer, MAPK signaling, drug resistance, tumor marker

\section{Introduction}

An organic anion-binding protein that functions to extract anions from the blood into hepatocytes was first found in the livers of rats and named Y protein or ligandin. ${ }^{1,2)}$ This protein was later shown to exert its enzymatic activity by conjugation of the reduced form of glutathione (GSH) to xenobiotic substrates for the purpose of detoxification, which is a characteristic of a phase II metabolic enzyme. Thereafter, similar enzymes with the same catalytic activity but distinct structures, i.e., isozymes, were reported, and these were categorized as a family of

*1 Oncology Section, Center of Advanced Medicine, Shonan Kamakura Innovation Park, Shonan Kamakura General Hospital, Kamakura, Kanagawa, Japan.

*2 Sapporo Medical University, Sapporo, Hokkaido, Japan.

*3 Department of Community Medicine for Gastroenterology and Oncology, Tokushima University Graduate School of Biomedical Sciences, Tokushima, Japan.

$\dagger \quad$ Correspondence should be addressed: Y. Niitsu, Oncology Section, Center of Advanced Medicine, Shonan Kamakura Innovation Park, Shonan Kamakura General Hospital, 1370-1 Okamoto, Kamakura, Kanagawa 247-8533, Japan (e-mail: y_ niitu@shonankamakura.or.jp).
glutathione-S transferases (GSTs). ${ }^{3)}$ Amongst these GST isozymes, glutathione S transferase P1 (GSTP1) has a unique feature in that it is highly expressed in malignant tissues and thus has been used clinically as tumor marker. ${ }^{4)}$ Furthermore, because certain types of anticancer drugs such as alkylating agents are readily detoxified by conjugation with GSH, malignant tissues expressing high levels of GSTP1 often exhibit resistance against these anticancer drugs. ${ }^{5)}$ To overcome this GSTP1-related drug resistance, inhibitors of the enzyme activity of GSTP1 have been developed and used clinically with some successful outcomes. ${ }^{6}$ Another characteristic feature of this enzyme is its chaperone activity with various intracellular proteins, including some signaling factors. The most notable characteristic of GSTP1 as a chaperone is that it negatively regulates the activity of JNK, which promotes apoptosis of cells, upon exposure to $\mathrm{H}_{2} \mathrm{O}_{2}$ or UV irradiation. ${ }^{7)}$ Assuming the regulation of JNK by GSTP1 as underlying molecular mechanism, a series of studies have exploited the possible involvement of GSTP1 in cancer cell growth, and indeed these have shown the cancer growth promoting activity of GSTP1 by both in vitro cell 
proliferation assays and in vivo tumorigenesis assays of xenografted cancer cells. ${ }^{8)}$ Independently from these studies, we determined a novel function of GSTP1 in promoting the growth of cancers such as mutated KRAS (mKRAS) and mutated BRAF (mBRAF), of which mitogen-activated protein kinase (MAPK) forms the primary signaling pathway. ${ }^{9)}$ The proposed function is based on the findings that in these cancers GSTP1 is induced by MAPK signaling and in turn interacts with CRAF to form a CRAF/ GSTP1 complex, which augments downstream signals of MAPK in an autocrine loop. We further revealed the fact that this autocrine loop signal was active independently from authentic signaling pathway, thereby any means of inhibiting authentic MAPK signal components such as siRNA KRAS, siRNA BRAF, or mBRAF inhibitors did not affect the loop signal, resulting in the tumor becoming refractory to treatment with MAPK inhibitors.

In this review, we will first briefly summarize malignancy-related studies on GSTP1, then discuss the GSTP1-dependent autocrine signal loop in mKRAS and mBRAF cancers, and finally propose a putative approach to inhibit the autocrine signal loop targeting the GSTP1/CRAF complex.

\section{Personal history (Y.N.) of GST research}

It was 1972-1973 when I started my researcher career as a postdoctoral fellow with Dr. Irving Listowsky at the Department of Biochemistry, Albert Einstein College of Medicine, NY.

At that time, our efforts were mainly focused on exploration of the secondary structure of the iron storage protein ferritin using circular dichroism (CD), which was his specialty as an organic chemist (Fig. 1). In parallel with CD experiments, I was looking for a more clinically relevant area, and luckily an opportunity came up with a project to develop an assay system to measure circulating ferritin, which is nowadays widely used as an essential marker to assess storage iron in the body. Our laboratory happened to be located in the building next to the Liver Center, of which director was Dr. Irwin Arias, currently a Senior Scientist, Emeritus at National Institutes of Health, who had just found a novel organic anion binding protein, ligandin in rat hepatocytes. A collaboration with Dr. Listowsky was formed to explore the molecular basis of interactions between various organic anions and ligandin through the application of CD. This was my first encounters with ligandin, which was later designated as glutathione-S transferase A1-1

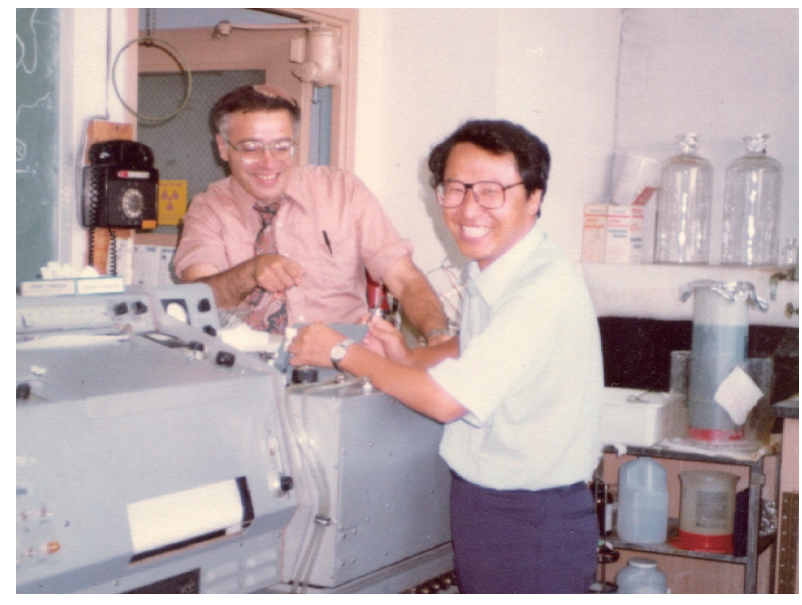

Fig. 1. Dr. Irving Listowsky and I (Y.N.) in front of the circular dichroism machine, in early 1972.

(GSTA1-1), ${ }^{2)}$ one of the GST isozymes, because the protein was revealed to exert an enzymatic activity to conjugate glutathione (GSH) to xenobiotics.

Since then, although "GST" has been a latent research interest, it took several years until restarting the GSTP1 project because at the Department of Medicine, Sapporo Medical University, Japan, our main research projects were rather clinically oriented, including iron depletion therapy for chronic hepatitis, ${ }^{10)}$ identification of microscopic precancerous lesions in the human colon using a magnifying endoscope, ${ }^{11)}$ treatment of minimal residual disease of acute myelogenous leukemia using an anti-VLA4 antibody ${ }^{12)}$ gene therapy for cancer, and the treatment of liver cirrhosis with siRNA HSP47 bearing vitamin A-coupled liposome, ${ }^{13)}$ amongst other topics.

While conducting these projects, I happened to come across publications on malignancy-related studies of GSTP1, which refreshed my research interest. Thereafter, we were fortunately able to produce a series of clinically relevant outcomes from the GSTP1 research as described below. It was also fortunate for me that Dr. Listowsky generously continued to support my projects from the viewpoint of basic science ever since I left his laboratory. Responding to his kindness, I have sent more than 40 scientists either to his laboratory or to the Liver Center of Einstein College, and one of those scientists, Dr. Tetsuji Takayama who is the coauthor of this article, came back full of GST knowledge and became closely engaged with our later GSTP1 research. This warm and close friendship between us and Dr. Listowsky lasted until the last moment, 
which came suddenly just few months ago (on April 29, 2021) when he passed away from COPD.

Therefore, I dedicate this review on GSTP1 to Dr. Listowsky in his memory.

\section{Structure and function of GSTs}

Most GST classes show a high degree of polymorphism, consisting of three superfamilies: cytosolic, mitochondrial, and microsomal-also known as MAPEG - proteins. ${ }^{14)}$ Amongst, the cytosolic family is the most well-characterized GSTs, and these are further divided into 13 sub-classes based upon their distinct structures, with nearly $40 \%$ sequence homology. ${ }^{15)}$

GSTs are usually composed of two identical subunits, each consisting of 199-244 amino acids, equivalent to $22-29 \mathrm{kDa}$, and containing two catalytically independent active sites, with the "G" site at the N-terminus and the "H" site in the C-terminal domain. With these two sites, GSTs exert the activity of a phase II metabolic enzyme, catalyzing the conjugation of GSH to xenobiotic substrates for the purpose of water-solubilization and detoxification. ${ }^{16), 17)}$

In addition to the conjugation reactions, GSTs have a role in several other catalytic activities, including thiol transferase, which catalyzes S-glutathionylation of proteins, and glutathione peroxidase, which catalyzes the reduction of reactive oxygen species and organic hydroperoxides such as phospholipids, fatty acids, and DNA hydroperoxides. ${ }^{18)}$

Notably, GSTs also exert non-enzymatic activities such as chaperone activities to modulate the function of various intracellular proteins. Amongst these, GSTP1 function as a chaperone to JNK, implying a role in cell survival and apoptosis as a member of the MAPK pathway, has been explored extensively. When cells are in a nonstress state, GSTP1 binds to JNK inhibiting its activity. ${ }^{8)}$

UV irradiation or $\mathrm{H}_{2} \mathrm{O}_{2}$ treatment caused dissociation of GSTP1 from JNK, in turn leading to JUN phosphorylation followed by the induction of AP-1-dependent target genes involved in cell proliferation, DNA repair, and cell death. ${ }^{19)}$

Another chaperone activity of GSTP1 to inhibit the function of target molecules is exhibited on tumor necrosis factor receptor-associated factor 2 (TRAF2), an upstream activator of JNK. ${ }^{20)}$ Thus, GSTP1 blocks the MAPK/JNK signaling cascade at multiple steps.

Completely opposite to the chaperone function of GSTP1, activation of glyceraldehyde-3-phosphate dehydrogenase, which plays an essential role in the metabolism and signaling pathway of triple negative breast cancer was reported recently. ${ }^{21)}$ Similar GSTP1-related activation of target molecule, peroxiredoxin 6 , of which the peroxidase activity protects cells against oxidant stress through GSH-dependent reduction of various hydroperoxides including phospholipid hydroperoxides to the corresponding water and alcohols, was also reported. ${ }^{22)}$

We also recently reported that GSTP1 binds to and activates CRAF and its downstream MAPK signaling in cancers with mutated KRAS or $\mathrm{BRAF}^{9}$ — - these results will be described in following sections. Incidentally, there is a possibility that, although not related to the chaperone function, the above-mentioned thiol transferase activity of GSTP1 plays a role in MAPK signaling, because self-Sglutathionylation of GSTP1 resulted in interference with GSTP1/JNK complex formation. ${ }^{7)}$ Passive activation of redox regulation of GSTP1 by binding to Fanconi anemia group C protein (FANCC) was also reported to be essential for the survival of hematopoietic progenitor cells. ${ }^{23)}$

\section{GSTP1 and malignancy}

GSTP1 is categorized as one of the 13 subclasses of cytosolic GSTs ${ }^{3)}$ and is representative of other members of the cytosolic GST superfamily as the first GST to have its structure determined. ${ }^{24)}$ One of the unique characteristics of GSTP1 compared with other cytosolic GSTs is its close association with malignancy. ${ }^{5), 25)}$ Because of this uniqueness, numerous studies on the clinical implication of GSTP1 such as its application as a tumor marker in the circulation and role as a chemo-resistant factor in malignant tissues have been conducted.

\section{GSTP1 as a tumor marker}

Elevated GSTP1 in the circulation has been reported in patients with various malignancies and has been proposed as a tumor marker for decades. ${ }^{18)}$ We reported the elevation of plasma GSTP1 in patients with gastrointestinal cancers, ${ }^{4)}$ oral cancers $^{26)}$ and malignant lymphoma. ${ }^{27)}$ However, GSTP1 is not a tumor-specific marker because it is widely distributed in normal tissues ${ }^{25)}$ and is released from these tissues upon damage such as in chronic kidney disease, liver disease, etc. ${ }^{28)}$ thus it may not be valid for the primary diagnosis of malignant diseases. Therefore, attempts, including ours, ${ }^{27)}$ have been made to utilize serum GSTP1 for determination of the stage of cancer or monitoring the efficacy of 
treatment, as with most other tumor-associated markers. However, contrary to these previous findings, in some malignancies such as esophageal squamous carcinoma, a correlation between decreased GSTP1 expression and poor prognosis has been reported. ${ }^{18), 29)}$ In addition, more recent interest in the use of GSTP1 as a tumor marker was focused on its methylation status. ${ }^{18)}$ In particular, in prostate cancer, hypermethylation of GSTP1 has been shown to be a useful marker for early detection. ${ }^{18), 30)}$ Similarly, GSTP1 hypermethylation was described as an early event in breast cancer. ${ }^{18), 31)}$ In some studies on hepatocellular cancer, a correlation between poor prognosis and hypermethylation was shown. ${ }^{18), 32)}$ Furthermore, application of liquid biopsy methods to detect GSTP1 hypermethylation in urine, serum, plasma, and blood from patients with cancer have been attempted, ${ }^{33)}$ although validation of this prognostic approach needs further clinical studies.

The mechanism of release of the cytoplasmic GSTP1 protein into the circulation is presently unknown. However, our previous observation that only the monomer form GSTP1 existed in the culture medium of platelets that contained abundant dimeric GSTP1,34) was suggestive of a selective secretion process of cytoplasmic GSTP1 into the circulation in the form of a monomer. Consistent with this assumption was that circulating GSTP1 was associated with exosomes, which were actively secreted from GSTP1-expressing tissues. ${ }^{35)}$ Because exosomes are known to be intercellular communication vectors from the original tissue to distant organs, it may be possibility that the function of GSTP1 can be transferred to target tissues. In fact, conferring drugresistant properties with GSTP1 by exosomes has been reported. ${ }^{35)}$

In this regard, the functions of the GSTP1 dimer and monomer forms are distinct from each other: dimer GSTP1 functions as an enzyme and monomer GSTP1 functions as a chaperone for some intracellular proteins. Therefore, it may also be interesting to examine which form of GSTP1 is present in exosomes for the possible transfer of functions to target tissues.

Another future task is to investigate whether plasma GSTP1 can be used as a tumor marker not only for cancers from certain designated organs but also for cancers caused by specific oncogenes such as mutated KRAS and BRAF, because in cancers caused by these oncogenes GSTP1 is induced through MAPK signaling and functions as a growth promoting factor. Details of this will be discussed in the following section. Hence, for monitoring the effect of therapeutic agents in these cancers, plasma GSTP1 levels should serve a reasonable marker.

\section{GSTP1 as a chemo-resistant factor}

Another clinically important issue regarding the association of GSTP1 with malignancy is its role in the development of resistance against drugs such as adriamycin (ADR), cisplatin (CDDP), melphalan, cyclophosphamide, and chlorambucil through the GSH-conjugation activity of GSTP1.5) We confirmed a significant increase in the sensitivity of a GSTP1expressing colon cell line to ADR, CDDP, melphalan, and etoposide by transfection of GSTP1 antisense DNA. ${ }^{36)}$ We also demonstrated that CD34 ${ }^{+}$bone marrow cells were protected from alkylating agents by introduction of the GSTP1 gene. ${ }^{37)}$ With regard to the reactive oxygen species (ROS) scavenging activity of GST (GSTP1), it may be intriguing to examine the role of GSTP1 in the chemoresistance of cancer stem cells in which a low ROS level is an essential factor for their stemness and chemoresistance properties. ${ }^{38)}$

\section{GSTP1 inhibitors}

To overcome GSH-conjugation-related refractory tumors, various drugs to inhibit the enzyme activity of GSTP1, including synthetic inhibitors ${ }^{39}$ ) and natural inhibitors from plants, ${ }^{40}$ ) have been explored. Amongst these, ethacrynic acid (EA), which has been used clinically as a diuretic drug, is the most extensively studied to overcome the GSTP1-refractory behavior in a wide range of malignancies. ${ }^{39)}$ The mechanism for the restoration of drug sensitivity by EA involves covalent binding of EA to GSH, thereby GSH becomes unavailable for conjugation to drugs by GSTP1. However, despite the fairly acceptable pharmacological effect on GSTP1-positive cancers, its clinical use was limited due to its strong diuretic properties. To avoid the unfavorable diuretic effect, various EA analogues have been developed.

Notably, most of these EA analogues exhibit anticancer activity in addition to their originally aimed activity to overcome drug refractoriness. ${ }^{41)}$ In particular, ethacraplatin, which is a cisplatin molecule coordinated to two ethacrynic acid ligands, may be a promising EA analogue because it was shown to be highly potent in treating cisplatin-resistant cancer cells. ${ }^{42)}$ Clinical trials on some of these EA analogues are currently underway. 
Another series of anti-GSTP1 drugs comprise GST analogues, of which the best characterized example is a GSH peptidomimetic molecule designated as ezatiostat hydrochloride (TLK199) whose metabolites bind the G-site of GSTP1 to inhibit its enzyme activity. This compound was proven to be clinically efficacious in the treatment of myelodysplastic syndrome ${ }^{43}$ ) although the mechanism of action is unclear. Canfosfamide, also known as TLK286, is also a GSH analogue that is activated by GSTP1-1 into a GSH derivative and phosphorodiamidate, an alkylating metabolite that forms covalent linkages with DNA, thereby exerting anticancer activity in ovarian cancer. ${ }^{44)}$

By modifying TLK199, we developed a new compound, O-hexadecyl- $\gamma$-glutamyl-S-benzyl-cysteinyl-D-phenyl glycine-ethylester (HGBPE), with higher solubility than the original drug, and demonstrated a significantly enhanced antitumor activity of ADR or cyclophosphamide against cholangiocarcinoma through combination use with a new compound in a xenograft model. ${ }^{45)}$ Its clinical efficacy needs to be examined in a future study.

The non-enzymatic activity of GSTP1, namely its chaperone function, also plays a critical role in the acquisition of drug resistance. In this regard, it may be worth mentioning that 6-(7-nitro-2,1,3-benzoxadiazol-4-ylthio) hexanol (NBDHEX) and its analogue MC3181, a derivative that binds to GSTP1 and dissociate from its complexes with both JNK and TRAF $2{ }^{46)}$ demonstrated strong blocking activity on cancer growth and metastasis in a xenograft mouse model of vemurafenib-resistant melanoma. ${ }^{47}$ Although the precise molecular mechanism is still under investigation, we found recently that NPD 926, which specifically binds to GSTP1 and evokes suppression of mKRAS cell growth, ${ }^{48)}$ overcomes vemurafenib resistance in melanoma cells, most likely by inhibiting the chaperone activity of GSTP1 (unpublished data). Details of this role of GSTP1 as a chaperone-related drug resistance factor will be discussed in the paragraphs bellow. Table 1 summarizes the anti-GSTP1 agents that may be used to overcome chemoresistance and also to treat cancer.

\section{Mechanisms underlying the elevation of GSTP1 in malignant tissues}

In the 1980s, an AP-1 response element was identified in the promoter region of the human GSTP1 gene by functional studies, and an absolute requirement for the AP-1 recognition sequence for transcriptional activity of the GSTP1 promoter was
Table 1. GSTP1 inhibitors

\begin{tabular}{|c|c|c|}
\hline $\begin{array}{l}\text { Ethacrynic acid } \\
\text { and analogues }\end{array}$ & Function & References \\
\hline Ethacrynic acid (EA) & $\begin{array}{l}\text { Diuretics } \\
\text { Overcoming drug resistance }\end{array}$ & 39 \\
\hline Other EA analogues & $\begin{array}{l}\text { Overcoming drug resistance } \\
\text { Anti-cancer activity }\end{array}$ & 41 \\
\hline Ethacraplatin & Anti-cancer activity & 42 \\
\hline Piperlongumine & $\begin{array}{l}\text { Alkaloid (plant) } \\
\text { Anti-cancer activity }\end{array}$ & 40 \\
\hline \multicolumn{3}{|l|}{ GSH analogues } \\
\hline TLK199 & $\begin{array}{l}\text { Overcoming drug resistance } \\
\text { Drug for myelodysplastic } \\
\text { syndrome }\end{array}$ & 43 \\
\hline $\begin{array}{l}\text { TLK286 } \\
\text { (Canfosfamide) } \\
\end{array}$ & $\begin{array}{l}\text { Anti-cancer activity for } \\
\text { ovarian cancer }\end{array}$ & 44 \\
\hline HGBPE & $\begin{array}{l}\text { Chemoprevention of pan- } \\
\text { creas cancer } \\
\text { Overcoming drug resistance }\end{array}$ & 45 \\
\hline $\begin{array}{l}\text { NBDHEX and its } \\
\text { analogue }\end{array}$ & $\begin{array}{l}\text { Chaperone activity of } \\
\text { GSTP1 to block its interac- } \\
\text { tion with JNK and TRAF2 } \\
\text { Overcoming mBRAF inhib- } \\
\text { itor resistance }\end{array}$ & 46,47 \\
\hline NPD926 & $\begin{array}{l}\text { Anti-cancer activity for } \\
\text { mKRAS cancer }\end{array}$ & 48 \\
\hline
\end{tabular}

also revealed. ${ }^{49), 50)}$ Subsequently, AP-1 as a transcription factor was found to be comprised of members of the Jun and Fos protein families. ${ }^{51)}$

Independently from these studies, we have been noticing a close relationship between the overexpression of GSTP1 and KRAS mutation. ${ }^{52)}$ For example, almost identical immunostaining patterns of GSTP1 with those of mKRAS were found not only in mKRAS-positive adenomas but also in aberrant crypt foci $(\mathrm{ACF}){ }^{53)}$ which we identified for the first time as microscopic mKRAS-positive precursor lesions of colon adenoma/cancer in the human colon (Fig. 2). ${ }^{11)}$ These findings led us to investigate a causal relationship between GSTP1 and mKRAS, and employing several mKRAS cell lines we confirmed that GSTP1 is indeed induced by binding of the end product of MAPK signaling transduced by mKRAS, AP-1, to TRE promotor of GSTP1 (Fig. 3). ${ }^{9)}$ Thus, we concluded that upregulation of GSTP1 expression in cancers with mutated KRAS, of 


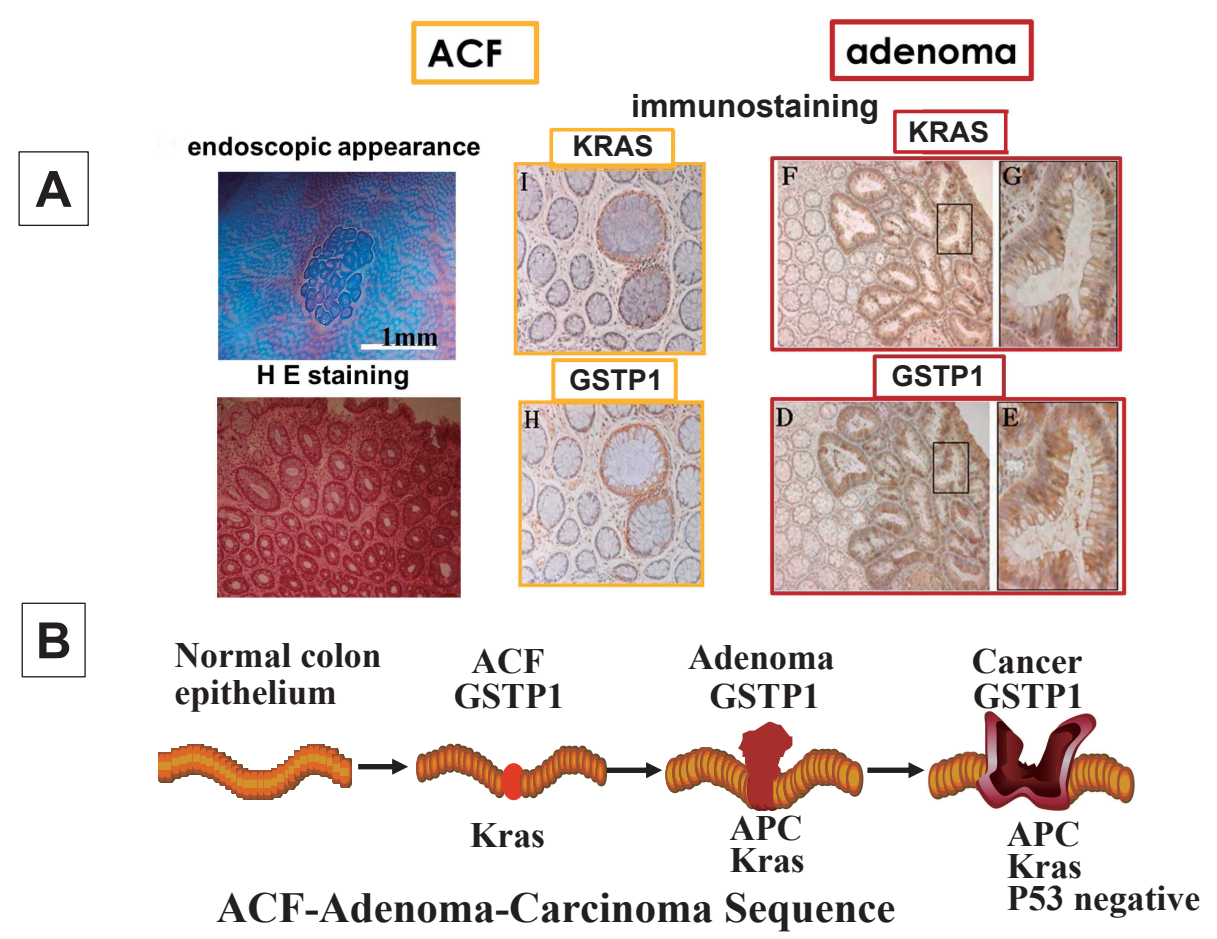

Fig. 2. Close relationship between GSTP1 expression and KRAS mutation during human colon carcinogenesis. Methylene blue-stained aberrant crypt foci (ACF) identified using a magnifying endoscope in the human colon and their appearance in an HE-stained specimen (left most panels of A). Immunostaining appearance of mutated KRAS and GSTP1 in human ACF (middle panels of A), immunostaining appearance of mutated KRAS and GSTP1 in human colon adenoma (right most panels of A). B) Scematic presentation of ACF-adenoma-carcinoma sequence. A close association of GSTP1 expression and Kras mutation is emphasized. Copyright of Fig. 2A was permitted from Gastroenterology (2001) 121, 865-874 (Ref. 52).

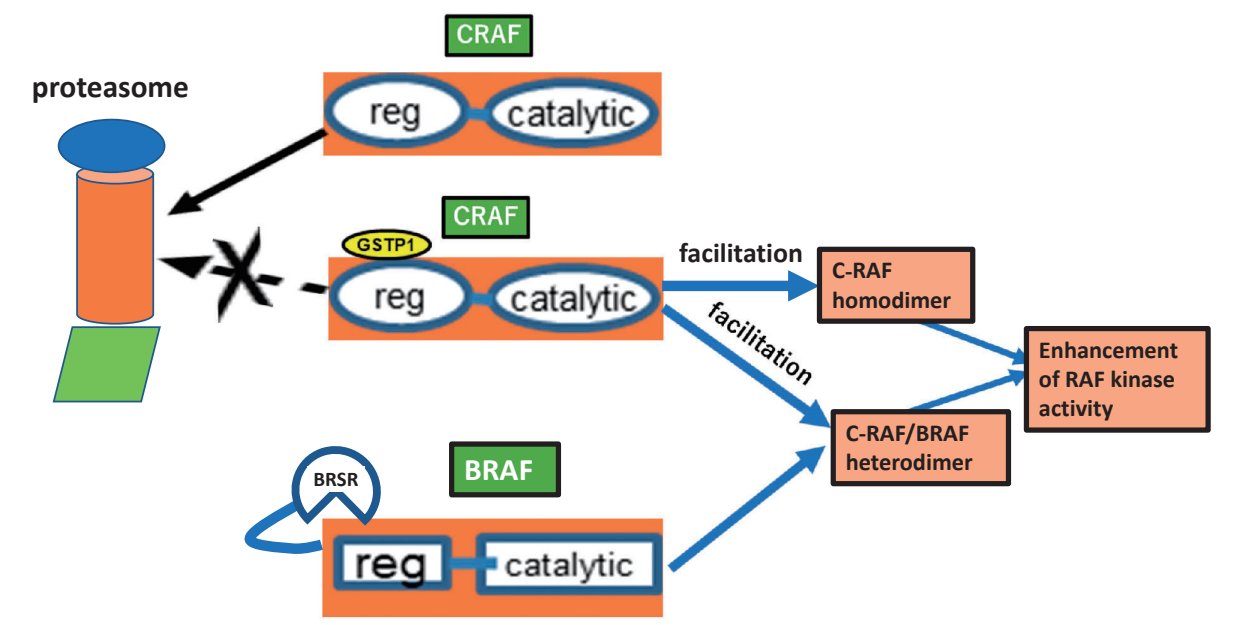

Fig. 3. Binding of GSTP1 to CRAF at its N-terminal domain, but not to BRAF, blocks proteasomal degradation of CRAF and facilitates dimerization of CRAF homodimer and CRAF/BRAF heterodimer giving rise to activation of these RAF dimers. The BRSR motif extending from the N-terminal domain of BRAF interferes with the binding of GSTP1 to BRAF.

which the main signal pathway is MAPK, is not a simple coincidental event but is a result of a causal relationship.

\section{Role of GSTP1 in cancer cell proliferation}

For the past 15 years, investigators have found 
that GSTP1 is involved in the proliferation of certain types of cancer cells, ${ }^{54)-56)}$ although the detailed molecular mechanisms underlying the growth promoting activity have not been explored but been simply ascribed, by speculation, to the chaperone activity of GSTP1 to JNK, as reported by Ronai et $a .^{7)}$

We, based on the aforementioned fact that GSTP1 expression is closely associated with KRAS mutation through the MAPK mediated induction, postulated that GSTP1 might be involved in the growth of mKRAS cancer. To address this, we transduced siRNA GSTP1 and GSTP1 expression viral vectors into mKRAS cells and non-GSTP1expressing cancer cells, respectively, and confirmed a significant growth suppression of the former cells and growth enhancement of the latter cells, verifying the growth promoting activity of GSTP1 in mKRAS cells. ${ }^{9)}$

With regard to the mechanism of this growth promoting activity, however, the regulation of JNK activity by GSTP1 was not apparent as far as mKRAS cells were concerned, because the growth and death rates of mKRAS cells in the presence of
JNK inhibitor were unchanged irrespective of siRNA GSTP1 treatment. ${ }^{9)}$

The chaperone activity of GSTP1 on CRAF, a signal component directly after mKRAS in the MAPK pathway, was unveiled because when we examined the MAPK signal components of mKRAS cells treated with siRNA GSTP1 or GSTP1 expression viral vector, decreased expression of p-CRAF / $\mathrm{CRAF} / \mathrm{p}-\mathrm{MEK} / \mathrm{P}-\mathrm{ERK}$ or increased expression of the same respective signal components were observed. ${ }^{9)}$

In a series of subsequent experiments, we showed that GSTP1 in mKRAS cells interacted with CRAF at its N-terminal domain to protect it from degradation by the ubiquitin-dependent proteasome pathway and to facilitate an essential step for signaling in the MAPK pathway involving the formation of RAF dimers; homodimer of CRAF and heterodimer of CRAF with BRAF (Fig. 4). ${ }^{9)}$

Because GSTP1, an end product of MAPK signaling, interacts with CRAF, an early signal component of the MAPK pathway, and enhances the signaling power, we designated this whole scheme as an "autocrine signal loop" (Fig. 4). Theoretically,

\section{A}

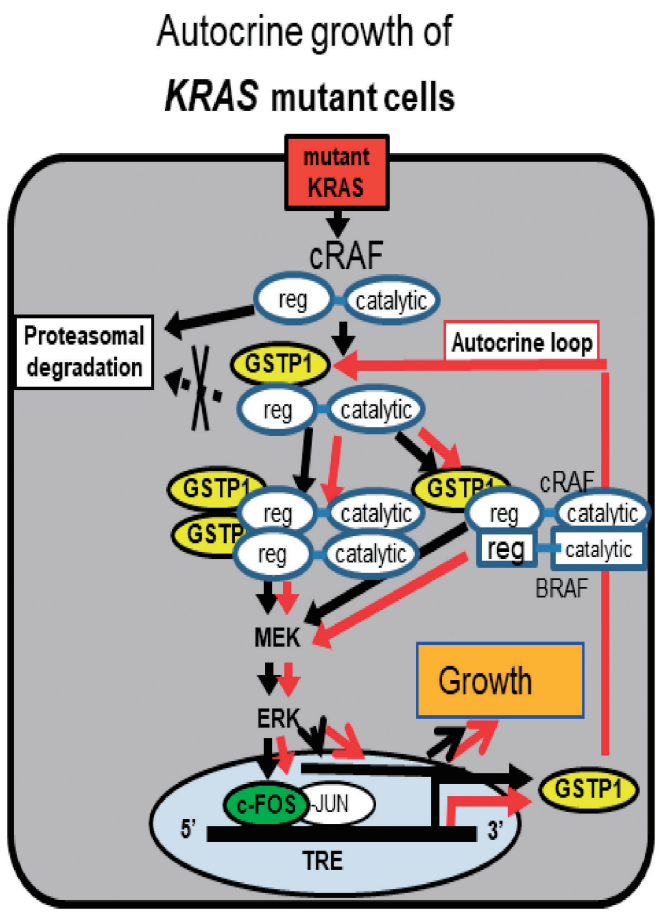

\section{B}

Autocrine growth of

BRAF(V600E) mutant cells

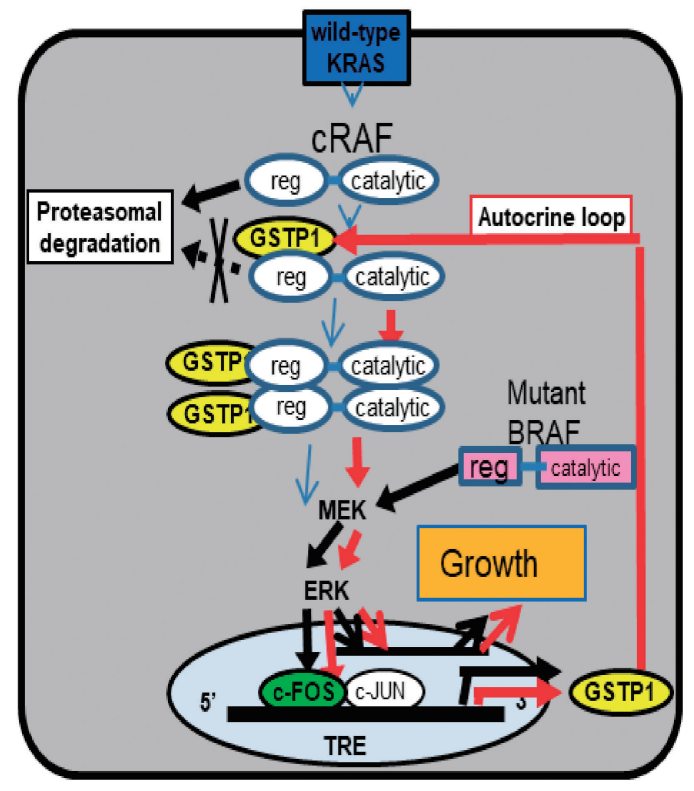

Fig. 4. Schematic presentation of autocrine signaling loops in mutated KRAS (A) and mutated BRAF (B) cells. In this diagram, the independent function of the autocrine signaling loop from authentic signals of oncogenes is highlighted. Copyright was permitted from PNAS (2020) 117, 19435-19445 (Ref. 9). 
this autocrine signal loop, once established, is considered to keep signaling as far as GSTP1 is present in the cells, irrespective of stimuli from mKRAS.

mBRAF cells also transduce growth signaling through the MAPK pathway; therefore, we examined the possibility that the end product of MAPK signaling, GSTP1, may be expressed in these cells as well, and as expected, confirmed the GSTP1 positivity in all mBRAF cells assessed. $\left.{ }^{9}\right)$

We then extended our exploration to see if the autocrine signal loop is also functioning in mBRAF cells and found significant growth suppression with impaired MAPK signaling in siRNA GSTP1-treated mBRAF cells (Fig. 4) ${ }^{9}{ }^{9}$ Hence, in cells with mKRAS, mBRAF and presumably with other oncogenes of which the primary signal pathway is MAPK, GSTP1 is deemed to play a critical role in their proliferation.

\section{GSTP1 and tumorigenesis}

The growth promoting activity of GSTP1 in cancer cells revealed by in vitro experiments has been further substantiated by investigators who demonstrated impaired tumor formation, tumorigenesis, of GSTP1-positive cancer cells xenografted into mice after silencing of GSTP1. ${ }^{54)}$ In these studies, however, the relationship between overexpression of GSTP1 and its causative oncogene was not elucidated, and the underlying mechanism for the GSTP1 activity was again speculatively ascribed to its chaperone function with JNK.

We also supported our in vitro findings that GSTP1 plays a critical role in the proliferation of mKRAS cells, using in vivo tumorigenesis experiments which showed, upon induction of siRNA GSTP1 by administration of Dox, significant suppression of tumor size in mice xenografted with mKRAS cells carrying doxycycline (Dox)-inducible shRNA GSTP1. ${ }^{9)}$ In addition, the suppression of tumor size was concomitantly associated with suppression of the GSTP1/CRAF complex, consistent with the results of in vitro findings of siRNA GSTP1treated mKRAS cells, confirming a role of GSTP1 as a chaperone protein with CRAF using tumorigenesis experiment.

\section{GSTP1 and carcinogenesis}

Studies on tumorigenesis are clinically important because they ultimately link to therapeutic strategies. Likewise, studies on carcinogenesis are clinically meaningful because they should provide clues for chemoprevention of the disease. Therefore, we

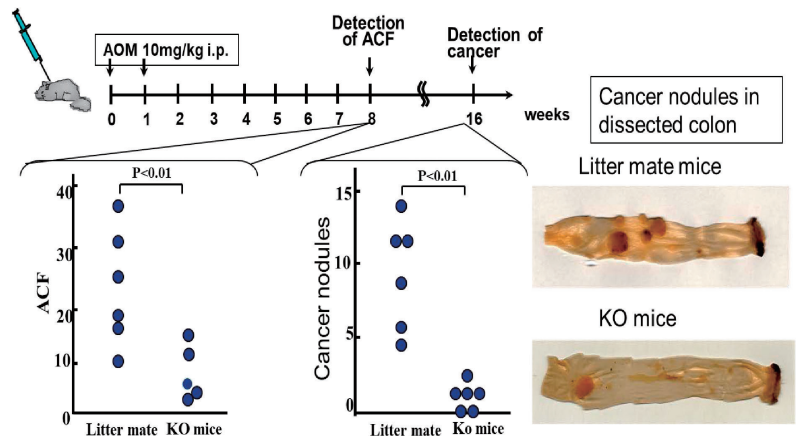

Fig. 5. Critical role of GSTP1 in mutated KRAS-positive colon carcinogenesis of azoxymethane-treated GSTP1 null mice. Note that formation of both ACF and cancer nodules shown in the left panels and cancer nodules in the right pictures were significantly impaired in GSTP1 null mice.

examined the role of GSTP1 in the carcinogenesis of mKRAS cancer. Practically, we employed GSTP1 null mice to follow the formation of $\mathrm{ACF}$ and subsequent cancerous polyps after administration of azoxymethane (AOM), which induces KRAS mutation in the colon epithelium. Compared with littermate mice that also received AOM, the numbers of $\mathrm{ACF}$ and cancerous polyps in GSTP1 null mice were substantially reduced (Fig. 5), $\left.{ }^{9}\right)$ indicating that without GSTP1 expression, mKRAS-dependent colon carcinogenesis may be significantly impaired and, therefore, chemoprevention approaches using antiGSTP1 agents should be highly efficacious for mKRAS colon cancer. Taking this into account, we examined the effect of a GSTP1 inhibitor, which we synthesized, HGBPE, on carcinogenesis in the pancreas in a murine model induced by 7,12dimethyl-benzanthracene (DMBA) and confirmed significant suppression of both precancerous lesions, ductal complexes, and adenocarcinoma, ${ }^{57)}$ proving a concept of chemoprevention of mKRAS cancer by inhibitor of GSTP1.

Clinical trials of cancer chemoprevention have been conducted for decades. Some of the most extensively studied trials are those on colon cancer using non-steroidal anti-inflammatory drugs (NSAIDs), which mostly generated promising outcomes. However, in these trials, drugs were administered for years or at least months to cancer-free subjects, causing low compliance and unexpected adverse effects. To avoid these drawbacks, we performed a trial with short-term (2 months) administration of sulindac to healthy subjects and colon adenoma patients who underwent polypectomy, examining ACF as surrogate markers and 
successfully assured the efficacy of the treatment in preventing adenoma formation in both groups, and suggested a periodic administration protocol of once every 2 years. ${ }^{58)}$ Similarly, we, considering the fact that mKRAS is the critical driver oncogene for pancreatic cancer, against which therapeutic efficacy by any current modalities is very limited and prevention is highly desired. Therefore, we conducted a clinical trial of short-term sulindac administration in patients with precursor lesions of pancreas cancer, branch duct-intraductal papillary mucinous neoplasms (BD-IPMNs), with promising outcomes. ${ }^{59)}$ The mechanism underlying the activity of NSAIDs is thought to be a suppression of prostaglandin E2 synthesis via cyclooxygenase-2 (Cox2) inhibition, which causes a decrease in cell proliferation and increases apoptosis. However, when we examined the expression of Cox2 and GSTP1 in ACF, it was generally negative for the former and positive for the latter. Furthermore, the BD-IPMN lesions were also strongly positive for GSTP1 expression but faintly positive or negative for Cox2 expression. These results indicated that the target molecule of sulindac in these lesions may not be Cox2 but GSTP1. Taken together, both experimental and clinical results were suggestive of using GSTP1 inhibitors as chemoprevention drugs for mKRAS cancers. However, as discussed above, because chemoprevention drugs are supposed to be administered to subjects without cancer for certain consecutive periods of time; at least few months even with the periodical short-term protocols, properties such as orally availability and lower toxicity are required. In this regard, the development of anti-GSTP1 drugs with such properties in the future will make chemoprevention of mKRAS cancers and possibly of mBRAF cancers more efficacious than currently available drugs.

Incidentally, there have been several previous studies on carcinogenesis in GSTP1 null mice demonstrating completely the opposite results to ours, such as high incidence of cancer development in the GSTP1 null mice. ${ }^{60), 61)}$ However, this controversy may be explained by the fact that the agents to induce cancers in these previous studies were, unlike our AOM, deemed to be inactivated by GSTP1, thereby in the absence of GSTP1 (GSTP1 null mice) cancers developed readily.

\section{Treatment of GSTP1-positive mKRAS and mBRAF cancers}

Cancers that develop in patients are, not like the precursors of cancer, generally complex and even heterogeneous in terms of genetic background, making them difficult to treat with a single agent. In addition, for mKRAS cancers, because of the very specific reason that the KRAS protein has a smooth surface and does not have any deep pockets that drugs can bind to, no efficacious inhibitor was available until novel drugs that irreversibly bind to mutant K-Ras $(\mathrm{G} 12 \mathrm{C})$, representing $11 \%$ of all mKRAS, were recently developed. ${ }^{62-64)}$ Even with these new drugs, efficacy rates on mKRAS-positive cancers in clinical trials were not as dramatic as initially expected, ${ }^{65), 66)}$ suggesting complexity in the growth signaling of mKRAS cancer and the necessity for combination therapy for this disease. In this regard, our findings that GSTP1 forms an autocrine signal loop that parallels authentic mKRAS signaling pathway may explain some aspects of the complexity.

In fact, although siRNAs against KRAS and GSTP1 gave rise to significant suppression of mKRAS cell proliferation, the combination of these siRNAs brought about clear additive effects with almost complete suppression, ${ }^{9)}$ indicating that control of the autocrine loop signal is required to achieve fully efficacious treatment of mKRAS cancers even after pan-mKRAS inhibitors become available in future.

Incidentally, for controlling the autocrine signal loop, simple silencing or the inhibition of GSTP1 itself may not be a satisfactory approach because, as mentioned above, GSTP1 interacts with several intracellular factors in addition to CRAF (Table 2), thus those intracellular factors may also be affected by silencing or inhibition of GSTP1, causing offtarget effects.

One of the strategies to avoid putative off-target effects may be interference of GSTP1 binding to CRAF, which takes place at the N-terminal domain

Table 2. GSTP1-interacting proteins

\begin{tabular}{llc}
\hline $\begin{array}{l}\text { Interacting } \\
\text { proteins }\end{array}$ & Function & References \\
\hline CRAF & Signal transduction & 9 \\
\hline JNK1 & Signal transduction & 8,19 \\
\hline TRAF2 & Signal transduction & 20 \\
\hline FANCC & $\begin{array}{l}\text { Protection of hematopoietic } \\
\text { cells }\end{array}$ & 23 \\
\hline G3PD (GAPDH) $)$ & $\begin{array}{l}\text { Regulator of cell metabolism in } \\
\text { triple negative breast cancer }\end{array}$ & 21 \\
\hline PRDX6 & Peroxidase (lipid etc.) & 22 \\
\hline
\end{tabular}




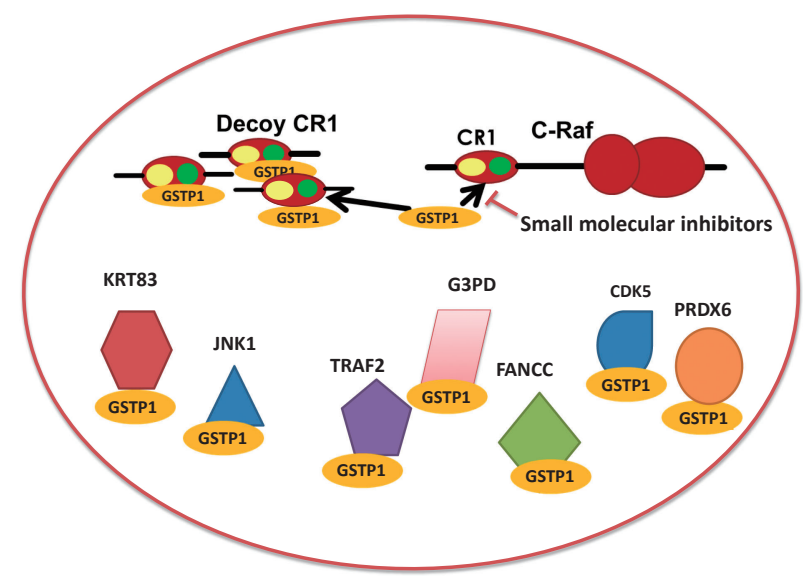

Fig. 6. Schematic presentation of putative approaches for specific blockade of interactions between GSTP1 and the CR1 motif (regulatory domain) of CRAF by CR1-decoy or small molecular inhibitors. Note that there are several GSTP1 binding molecules other than CRAF.

of CRAF (Fig. 3). Recent reports emphasize that inhibition of protein-protein interactions is becoming a realistic approach for controlling various biological cell behaviors, ${ }^{67)}$ and the development of inhibitor(s) of the GSTP1/CRAF interaction is certainly one option to assure the efficacy of combination therapy for mKRAS cancers. To validate this treatment concept, we have recently employed an expression vector of an N-terminal decoy of CRAF, the CR1motif, which should competitively block binding of GSTP1 to CRAF (Fig. 6), and succeeded in demonstrating the growth suppression of mKRAS cells transduced with this vector (unpublished observation). Based on this finding, we are currently working on a candidate small molecular compound that reduces CRAF expression by binding to GSTP1 (Fig. 6), expecting upcoming promising results.

Another concern for the treatment of mKRAS cancers is the fact that the negative feedback from p-ERK to early signaling components such as SOS, CRAF, MEK, and tyrosine kinase receptors is taking place in these cancers. ${ }^{68)-70)}$ Because there are no specific inhibitors to mKRASs except for K-Ras (G12C), a modality to inhibit lower MAPK signal components such as MEK is often chosen as alternative option to suppress mKRAS signaling. Therefore, the negative feedback may be suppressed by the MAPK inhibitor, ${ }^{71)}$ resulting in reactivation of early signaling components and acquisition of refractoriness to MAPK inhibitors. This reactivation of negative feedback was recently confirmed even with inhibitors for K-Ras(G12C). ${ }^{72)}$ In this regard, the autocrine signal loop in such mKRAS cells may also be enhanced when negative feedback is suppressed with MAPK inhibitors or K-Ras (G12C) inhibitors, particularly as CRAF, which is a counterpart of GSTP1 in the autocrine signaling loop, is one of the direct targets of p-ERK to be phosphorylated and downregulated in negative feedback signaling, ${ }^{70}$ ) though this GSTP1-related dilemma will also be managed when an approach to inhibit the GSTP1/ CRAF interaction become available.

The treatment strategy for mBRAF cells was deemed to be relatively straightforward compared with that for mKRAS cells because several mBRAFspecific inhibitors have been already developed. ${ }^{73)}$ In fact, first-generation inhibitors of the mBRAF monomer, which is active as oncogene by itself, while the active form of BRAF is generally a dimer, elicited remarkable efficacy in all initial trials, with apparently compatible results to the concept of "oncogenic addiction of cancer". ${ }^{74}$ However, because the autocrine loop signaling formed by GATP1/ CRAF complex is also active in mBRAF cells, independently from mBRAF monomer signaling, mBRAF monomer inhibitor alone is supposedly ineffective to bring about full inhibition of growth of mBRAF cancer. In fact, when we treated mBRAF cells with each mBRAF inhibitor and siRNA GSTP1, significant but not substantial growth suppression was observed. However, the combination of these two modalities caused almost complete growth suppression, ${ }^{9)}$ indicating that inhibition of the autocrine signaling loop in combination with mBRAF inhibitor is necessary to achieve greater efficacy in the treatment of mBRAF cancer as well. Furthermore, as the results of clinical studies on mBRAF-positive melanoma accumulated, frequent acquisition of resistance against the first-generation inhibitors has been reported. ${ }^{75), 76)}$ One of the mechanisms for this resistance was dimerization of $\mathrm{mBRAF}$; formation of either intact or truncated dimer molecules to which monomer-specific first-generation inhibitors were not effective. ${ }^{77), 78)}$ To overcome this type of resistance, second-generation inhibitors that inhibit both monomer and dimer mBRAFs were developed and introduced into clinical trials. ${ }^{78), 79)}$ Despite the quite promising outcomes of initial clinical trials of these inhibitors, there are still some cases that showed refractoriness to those inhibitors.

Mechanisms underlying such refractoriness to first- and second-generation inhibitors includes activation of co-existing insidious oncogenes such as 
mutated mKRAS, mutated EGFR, mutated MEK, mutated ARAF, etc., after suppression of the authentic signal by the mBRAF inhibitors. ${ }^{80,81)}$

In addition, similar mechanisms to that of mKRAS cancer treated with MAPK inhibitors involving activation of early signaling components of the MAPK pathway by suppression of negative feedback with mBRAF inhibitors, may underlie one of the mechanisms for the acquisition of resistance to mBRAF inhibitors. ${ }^{82}$ ) In order to overcome this resistance, co-administration of mBRAF inhibitors with inhibitors of early signaling components of MAPK such as MEK inhibitors has been attempted, although clinical trials were sometimes hampered by adverse effects. ${ }^{71}$ ) The autocrine signaling loop formed by the interaction of GSTP1 with CRAF is also implicated in the acquisition of resistance to mBRAF inhibitors.

We have most recently reported that by continuous exposure to one of the first generation of mBRAF inhibitors, melanoma cells with a V600E point mutation in BRAF acquired resistance to this inhibitor, and the resistance was overcome by siRNA GSTP1 treatment (unpublished observation), indicating GSTP1-induced resistance. Hence, the development of small molecular inhibitors of GSTP1/ CRAF interactions as mentioned above, is urgently needed not only for achieving efficacious treatment but also for overcoming drug-refractoriness in mBRAF cancers.

\section{Conclusion}

In the early 2000s, the concept of "oncogene addiction", which implies that the growth and survival of cancer cells can be impaired by the inactivation of a single oncogene, was proposed. However, as more sophisticated techniques in molecular biology have been introduced for the analysis of cancer cell signaling, concealed complexities of the signal pathways transduced by oncogenes have been revealed. The complexity often causes refractoriness to treatment with single oncogene inhibition. In particular, the unexpected emergence of new signal pathway(s) after treatment with oncogene-specific inhibitors is problematic because they often cause drug resistance.

Here, we reviewed the role of GSTP1, which primarily functions in the detoxification of xenobiotics through GSH conjugation, in the growth signaling of mKRAS and mBRAF cells, and emphasizing its chaperone activity with CRAF to create an autocrine signaling loop, for which we recently reported its existence in these cells based on the accumulated experimental evidence over several years.

This autocrine signaling loop gives rise to the complexity of the signaling pathway of these cancer cells, and probably other cancer cells with MAPK signaling as well, causing not only insufficient efficacy of inhibitors against authentic signaling components but also the acquisition of drug resistance. To overcome the difficulties related to this autocrine signaling loop, we proposed a strategy to inhibit GSTP1/CRAF complex formation with a small molecular agent. Experiments to prove the efficacy of this agent are currently underway.

\section{Acknowledgement}

We are in debt to Dr. Nobuaki Shinozaki, president of Shonan Kamakura General Hospital for his continuous encouragement and financial support of experiments performed at the Oncology Section, Center of Advanced Medicine. We also thank Dr. Yasuyuki Tashiro, Mr. Wataru Kurata, Dr. Rei Shimoyama, and Ms. Yukimi Seo from the Oncology Section, Center of Advanced Medicine, Shonan Kamakura Innovation Park, Shonan Kamakura General Hospital for their assistance with experiments on mKRAS and mBRAF cells. We further appreciate Mr. Akihiro Yokoyama and Mr. Wataru Yamazaki from Kamakura Roken, Kamakura, for their help in preparing this manuscript.

\section{References}

1) Reyes, H., Levi, A.J., Gatmaitan, Z. and Arias, I.M. (1969) Organic anion-binding protein in rat liver: drug induction and its physiologic consequence. Proc. Natl. Acad. Sci. U.S.A. 64, 168-170.

2) Litwack, G., Ketterer, B. and Arias, I.M. (1971) Ligandin: a hepatic protein which binds steroids, bilirubin, carcinogens and a number of exogenous organic anions. Nature 234, 466-467.

3) Mannervik, B., Board, P.G., Hayes, J.D., Listowsky, I. and Pearson, W.R. (2005) Nomenclature for mammalian soluble glutathione transferases. Methods Enzymol. 401, 1-8.

4) Niitsu, Y., Takahashi, Y., Saito, T., Hirata, Y., Arisato, N., Maruyama, H. et al. (1989) Serum glutathione-S-transferase-pi as a tumor marker for gastrointestinal malignancies. Cancer 63, 317323.

5) Townsend, D.M. and Tew, K.D. (2003) The role of glutathione- $S$-transferase in anti-cancer drug resistance. Oncogene 22, 7369-7375.

6) Vergote, I., Finkler, N.J., Hall, J.B., Melnyk, O., Edwards, R.P., Jones, M. et al. (2010) Randomized phase III study of canfosfamide in combination 
with pegylated liposomal doxorubicin compared with pegylated liposomal doxorubicin alone in platinum-resistant ovarian cancer. Int. J. Gynecol. Cancer 20, 772-780.

7) Adler, V., Yin, Z., Fuchs, S.Y., Benezra, M., Rosario, L., Tew, K.D. et al. (1999) Regulation of JNK signaling by GSTp. EMBO J. 18, 1321-1334.

8) Dang, D.T., Chen, F., Kohli, M., Rago, C., Cummins, J.M. and Dang, L.H. (2005) Glutathione S-transferase pil promotes tumorigenicity in HCT116 human colon cancer cells. Cancer Res. 65, 9485-9494.

9) Niitsu, Y., Sato, Y., Takanashi, K., Hayashi, T., Kubo-Birukawa, N., Shimizu, F. et al. (2020) A CRAF/glutathione-S-transferase P1 complex sustains autocrine growth of cancers with $K R A S$ and $B R A F$ mutations. Proc. Natl. Acad. Sci. U.S.A. 117, 19435-19445.

10) Kato, J., Kobune, M., Kohgo, Y., Sugawara, N., Hisai, H., Nakamura, T. et al. (1996) Hepatic iron deprivation prevents spontaneous development of fulminant hepatitis and liver cancer in Long-Evans Cinnamon rats. J. Clin. Invest. 98, 923-929.

11) Takayama, T., Katsuki, S., Takahashi, Y., Ohi, M., Nojiri, S., Sakamaki, S. et al. (1998) Aberrant crypt foci of the colon as precursors of adenoma and cancer. N. Engl. J. Med. 339, 1277-1284.

12) Matsunaga, T., Takemoto, N., Sato, T., Takimoto, R., Tanaka, I., Fujimi, A. et al. (2003) Interaction between leukemic-cell VLA-4 and stromal fibronectin is a decisive factor for minimal residual disease of acute myelogenous leukemia. Nat. Med. 9, 1158-1165, Erratum in: (2005) Nat. Med. 11, 578.

13) Sato, Y., Murase, K., Kato, J., Kobune, M., Sato, T., Kawano, Y. et al. (2008) Resolution of liver cirrhosis using vitamin A-coupled liposomes to deliver siRNA against a collagen-specific chaperone. Nat. Biotechnol. 26, 431-442.

14) Singh, S. (2015) Cytoprotective and regulatory functions of glutathione S-transferases in cancer cell proliferation and cell death. Cancer Chemother. Pharmacol. 75, 1-15.

15) Allocati, N., Masulli, M., Di Ilio, C. and Federici, L. (2018) Glutathione transferases: substrates, inihibitors and pro-drugs in cancer and neurodegenerative diseases. Oncogenesis $\mathbf{7}, 8$.

16) Prade, L., Huber, R., Manoharan, T.H., Fahl, W.E. and Reuter, W. (1997) Structures of class pi glutathione S-transferase from human placenta in complex with substrate, transition-state analogue and inhibitor. Structure 5, 1287-1295.

17) Tew, K.D. and Townsend, D.M. (2012) Glutathiones-transferases as determinants of cell survival and death. Antioxid. Redox Signal. 17, 1728-1737.

18) Cui, J., Li, G., Yin, J., Li, L., Tan, Y., Wei, H. et al. (2020) GSTP1 and cancer: expression, methylation, polymorphisms and signaling (Review). Int. J. Oncol. 56, 867-878.

19) Tew, K.D., Manevich, Y., Grek, C., Xiong, Y., Uys, J. and Townsend, D.M. (2011) The role of glutathione S-transferase $\mathrm{P}$ in signaling pathways and S-glutathionylation in cancer. Free Radic. Biol. Med. 51, 299-313.

20) Wu, Y., Fan, Y., Xue, B., Luo, L., Shen, J., Zhang, S. et al. (2006) Human glutathione S-transferase P1-1 interacts with TRAF2 and regulates TRAF2ASK1 signals. Oncogene 25, 5787-5800.

21) Louie, S.M., Grossman, E.A., Crawford, L.A., Ding, L., Camarda, R., Huffman, T.R. et al. (2016) GSTP1 is a driver of triple-negative breast cancer cell metabolism and pathogenicity. Cell Chem. Biol. 23, 567-578.

22) Zhou, S., Lien, Y.C., Shuvaeva, T., DeBolt, K., Feinstein, S.I. and Fisher, A.B. (2013) Functional interaction of glutathione S-transferase pi and peroxiredoxin 6 in intact cells. Int. J. Biochem. Cell Biol. 45, 401-407.

23) Cumming, R.C., Lightfoot, J., Beard, K., Youssoufian, H., O'Brien, P.J. and Buchwald, M. (2001) Fanconi anemia group C protein prevents apoptosis in hematopoietic cells through redox regulation of GSTP1. Nat. Med. 7, 814-820.

24) Oakley, A. (2011) Glutathione transferases: a structural perspective. Drug Metab. Rev. 43, 138151.

25) GSTP1 protein summary. The Human Protein Atlas, https://www.proteinatlas.org/ ENSG00000084207-GSTP1.

26) Hirata, S., Odajima, T., Kohama, G., Ishigaki, S. and Niitsu, Y. (1992) Significance of glutathione Stransferase-pi as a tumor marker in patients with oral cancer. Cancer 70, 2381-2387.

27) Katahira, T., Takayama, T., Miyanishi, K., Hayashi, T., Ikeda, T., Takahashi, Y. et al. (2004) Plasma glutathione S-Transferase P1-1 as a prognostic factor in patients with advanced non-Hodgkin's lymphoma (stages III and IV). Clin. Cancer Res. 10, 7934-7940.

28) Bocedi, A., Noce, A., Marrone, G., Noce, G., Cattani, G., Gambardella, G. et al. (2019) Glutathione transferase P1-1 an enzyme useful in biomedicine and as biomarker in clinical practice and in environmental pollution. Nutrients 11, 1741.

29) Wang, Z., He, W., Yang, G., Wang, J., Wang, Z., Nesland, J.M. et al. (2010) Decreased expression of GST pi is correlated with a poor prognosis in human esophageal squamous carcinoma. BMC Cancer 10, 352.

30) Martignano, F., Gurioli, G., Salvi, S., Calistri, D., Costantini, M., Gunelli, R. et al. (2016) GSTP1 methylation and protein expression in prostate cancer: diagnostic implications. Dis. Markers 2016, 4358292 .

31) Lee, J.S. (2007) GSTP1 promoter hypermethylation is an early event in breast carcinogenesis. Virchows Arch. 450, 637-642, Erratum in: (2007) Virchows Arch. 451, 741.

32) Li, Q.F., Li, Q.Y., Gao, A.R. and Shi, Q.F. (2015) Correlation between promoter methylation in the GSTP1 gene and hepatocellular carcinoma development: a meta-analysis. Genet. Mol. Res. 14, $6762-6772$. 
33) Gurioli, G., Martignano, F., Salvi, S., Costantini, M., Gunelli, R. and Casadio, V. (2018) GSTP1 methylation in cancer: a liquid biopsy biomarker? Clin. Chem. Lab. Med. 56, 702-717.

34) Kura, T., Takahashi, Y., Takayama, T., Ban, N., Saito, T., Kuga, T. et al. (1996) Glutathione Stransferase-pi is secreted as a monomer into human plasma by platelets and tumor cells. Biochim. Biophys. Acta 1292, 317-323.

35) Yang, S.J., Wang, D.D., Li, J., Xu, H.Z., Shen, H.Y., Chen, X. et al. (2017) Predictive role of GSTP1containing exosomes in chemotherapy-resistant breast cancer. Gene 623, 5-14.

36) Ban, N., Takahashi, Y., Takayama, T., Kura, T., Katahira, T., Sakamaki, S. et al. (1996) Transfection of glutathione S-transferase (GST)-pi antisense complementary DNA increases the sensitivity of a colon cancer cell line to adriamycin, cisplatin, melphalan, and etoposide. Cancer Res. 56, 3577-3582.

37) Kuga, T., Sakamaki, S., Matsunaga, T., Hirayama, Y., Kuroda, H., Takahashi, Y. et al. (1997) Fibronectin fragment-facilitated retroviral transfer of the glutathione-S-transferase pi gene into $\mathrm{CD} 4^{+}$cells to protect them against alkylating agents. Hum. Gene Ther. 8, 1901-1910.

38) Bigarella, C.L., Liang, R. and Ghaffari, S. (2014) Stem cells and the impact of ROS signaling. Development 141, 4206-4218.

39) Gaté, L. and Tew, K.D. (2001) Glutathione Stransferases as emerging therapeutic targets. Expert Opin. Ther. Targets 5, 477-489.

40) Harshbarger, W., Gondi, S., Ficarro, S.B., Hunter, J., Udayakumar, D., Gurbani, D. et al. (2017) Structural and biochemical analyses reveal the mechanism of glutathione $S$-transferase $\mathrm{Pi} 1$ inhibition by the anti-cancer compound piperlongumine. J. Biol. Chem. 292, 112-120.

41) Punganuru, S.R., Mostofa, A.G.M., Madala, H.R., Basak, D. and Srivenugopal, K.S. (2016) Potent anti-proliferative actions of a non-diuretic glucosamine derivative of ethacrynic acid. Bioorg. Med. Chem. Lett. 26, 2829-2833.

42) Ang, W.H., Pilet, S., Scopelliti, R., Bussy, F., Juillerat-Jeanneret, L. and Dyson, P.J. (2005) Synthesis and characterization of platinum(IV) anticancer drugs with functionalized aromatic carboxylate ligands: influence of the ligands on drug efficacies and uptake. J. Med. Chem. 48, 8060-8069.

43) Mahadevan, D. and Sutton, G.R. (2015) Ezatiostat hydrochloride for the treatment of myelodysplastic syndromes. Expert Opin. Investig. Drugs 24, 725733 .

44) Kavanagh, J.J., Levenback, C.F., Ramirez, P.T., Wolf, J.L., Moore, C.L., Jones, M.R. et al. (2010) Phase 2 study of canfosfamide in combination with pegylated liposomal doxorubicin in platinum and paclitaxel refractory or resistant epithelial ovarian cancer. J. Hematol. Oncol. 3, 9.

45) Nakajima, T., Takayama, T., Miyanishi, K., Nobuoka, A., Hayashi, T., Abe, T. et al. (2003)
Reversal of multiple drug resistance in cholangiocarcinoma by the glutathione S-transferase-pispecific inhibitor O1-hexadecyl-gamma-glutamylS-benzylcysteinyl-D-phenylglycine ethylester. J. Pharmacol. Exp. Ther. 306, 861-869.

46) Sau, A., Filomeni, G., Pezzola, S., D'Aguanno, S., Tregno, F.P., Urbani, A. et al. (2012) Targeting GSTP1-1 induces JNK activation and leads to apoptosis in cisplatin-sensitive and -resistant human osteosarcoma cell lines. Mol. Biosyst. 8, 9941006.

47) Graziani, G., Artuso, S., De Luca, A., Muzi, A., Rotili, D., Scimeca, M. et al. (2015) A new water soluble MAPK activator exerts antitumor activity in melanoma cells resistant to the BRAF inhibitor vemurafenib. Biochem. Pharmacol. 95, $16-27$.

48) Kawamura, T., Kondoh, Y., Muroi, M., Kawatani, M. and Osada, H. (2014) A small molecule that induces reactive oxygen species via cellular glutathione depletion. Biochem. J. 463, 53-63.

49) Morrow, C.S., Goldsmith, M.E. and Cowan, K.H. (1990) Regulation of human glutathione Stransferase pi gene transcription: influence of $5^{\prime}$ flanking sequences and trans-activating factors which recognize AP-1-binding sites. Gene $\mathbf{8 8}$, 215-225.

50) Okuda, A., Imagawa, M., Maeda, Y., Sakai, M. and Muramatsu, M. (1989) Structural and functional analysis of an enhancer GPEI having a phorbol 12-O-tetradecanoate 13 -acetate responsive element-like sequence found in the rat glutathione transferase P gene. J. Biol. Chem. 264, 1691916926 .

51) Moffat, G.J., McLaren, A.W. and Wolf, C.R. (1994) Involvement of Jun and Fos proteins in regulating transcriptional activation of the human pi class glutathione S-transferase gene in multidrug-resistant MCF7 breast cancer cells. J. Biol. Chem. 269, 16397-16402.

52) Miyanishi, K., Takayama, T., Ohi, M., Hayashi, T., Nobuoka, A., Nakajima, T. et al. (2001) Glutathione S-transferase-pi overexpression is closely associated with K-ras mutation during human colon carcinogenesis. Gastroenterology 121, 865874.

53) Takayama, T., Ohi, M., Hayashi, T., Miyanishi, K., Nobuoka, A., Nakajima, T. et al. (2001) Analysis of K-ras, APC, and beta-catenin in aberrant crypt foci in sporadic adenoma, cancer, and familial adenomatous polyposis. Gastroenterology 121, 599-611.

54) Dang, D.T., Chen, F., Kohli, M., Rago, C., Cummins, J.M. and Dang, L.H. (2005) Glutathione S-transferase pi1 promotes tumorigenicity in HCT116 human colon cancer cells. Cancer Res. 65, 9485-9494.

55) Hokaiwado, N., Takeshita, F., Naiki-Ito, A. Asamoto, M., Ochiya, T. and Shirai, T. (2008) Glutathione S-transferase Pi mediates proliferation of androgen-independent prostate cancer cells. Carcinogenesis 29, 1134-1138. 
56) Okamura, T., Antoun, G., Keir, S.T., Friedman, H., Bigner, D.D. and Ali-Osman, F. (2015) Phosphorylation of glutathione $S$-transferase P1 (GSTP1) by epidermal growth factor receptor (EGFR) promotes formation of the GSTP1-c-Jun N-terminal kinase (JNK) complex and suppresses JNK downstream signaling and apoptosis in brain tumor cells. J. Biol. Chem. 290, 30866-30878.

57) Kogawa, T., Sato, Y., Shimoyama, R., Kurata, W., Tashiro, Y. and Niitsu, Y. (2021) Chemoprevention of pancreatic cancer by inhibition of glutathione-S transferase P1. Invest. New Drugs 39, $1484-1492$.

58) Takayama, T., Nagashima, H., Maeda, M., Nojiri, S., Hirayama, M., Nakano, Y. et al. (2011) Randomized double-blind trial of sulindac and etodolac to eradicate aberrant crypt foci and to prevent sporadic colorectal polyps. Clin. Cancer Res. 17, 3803-3811.

59) Hayashi, T., Ishiwatari, H., Ihara, H., Kawano, Y., Takada, K., Miyanishi, K. et al. (2009) Suppressive effect of sulindac on branch duct-intraductal papillary mucinous neoplasms. J. Gastroenterol. 44, 964-975.

60) Henderson, C.J., Smith, A.G., Ure, J., Brown, K., Bacon, E.J. and Wolf, C.R. (1998) Increased skin tumorigenesis in mice lacking pi class glutathione S-transferases. Proc. Natl. Acad. Sci. U.S.A. 95, $5275-5280$

61) Ritchie, K.J., Henderson, C.J., Wang, X.J., Vassieva, O., Carrie, D., Farmer, P.B. et al. (2007) Glutathione transferase pi plays a critical role in the development of lung carcinogenesis following exposure to tobacco-related carcinogens and urethane. Cancer Res. 67, 9248-9257.

62) Lito, P., Solomon, M., Li, L.S., Hansen, R. and Rosen, N. (2016) Allele-specific inhibitors inactivate mutant KRAS G12C by a trapping mechanism. Science 351, 604-608.

63) Hallin, J., Engstrom, L.D., Hargis, L., Calinisan, A., Aranda, R., Briere, D.M. et al. (2020) The KRASG12C inhibitor MRTX849 provides insight $^{2}$ toward therapeutic susceptibility of KRAS-mutant cancers in mouse models and patients. Cancer Discov. 10, 54-71.

64) Hong, D.S., Fakih, M.G., Strickler, J.H., Desai, J., Durm, G.A., Shapiro, G.I. et al. (2020) KRASG12C inhibition with sotorasib in advanced solid tumors. N. Engl. J. Med. 383, 1207-1217.

65) Tanaka, N., Lin, J.J., Li, C., Ryan, M.B., Zhang, J., Kiedrowski, L.A. et al. (2021) Clinical acquired resistance to KRAS ${ }^{\mathrm{G} 12 \mathrm{C}}$ inhibition through a novel KRAS switch-II pocket mutation and polyclonal alterations converging on RAS-MAPK reactivation. Cancer Discov. 11, 1913-1922.

66) Awad, M.M., Liu, S., Rybkin, I.I., Arbour, K.C., Dilly, J., Zhu, V.W. et al. (2021) Acquired resistance to KRAS G12C inhibition in cancer. N. Engl. J. Med. 384, 2382-2393.

67) Lu, H., Zhou, Q., He, J., Jiang, Z., Peng, C., Tong, R. et al. (2020) Recent advances in the development of protein-protein interactions modulators: mech- anisms and clinical trials. Signal Transduct. Target Ther. 5, 213.

68) Lake, D., Corrêa, S.A. and Müller, J. (2016) Negative feedback regulation of the ERK1/2 MAPK pathway. Cell. Mol. Life Sci. 73, 4397-4413.

69) Kochańczyk, M., Kocieniewski, P., Kozłowska, E., Jaruszewicz-Błońska, J., Sparta, B., Pargett, M. et al. (2017) Relaxation oscillations and hierarchy of feedbacks in MAPK signaling. Sci. Rep. 7, 38244.

70) Dougherty, M.K., Müller, J., Ritt, D.A., Zhou, M., Zhou, X.Z., Copeland, T.D. et al. (2005) Regulation of Raf-1 by direct feedback phosphorylation. Mol. Cell 17, 215-224.

71) Kun, E., Tsang, Y.T.M., Ng, C.W., Gershenson, D.M. and Wong, K.K. (2021) MEK inhibitor resistance mechanisms and recent developments in combination trials. Cancer Treat. Rev. 92, 102137.

72) Ryan, M.B., Fece de la Cruz, F., Phat, S., Myers, D.T., Wong, E., Shahzade, H.A. et al. (2020) Vertical pathway inhibition overcomes adaptive feedback resistance to KRAS ${ }^{\mathrm{G} 12 \mathrm{C}}$ inhibition. Clin. Cancer Res. 26, 1633-1643.

73) Bollag, G., Hirth, P., Tsai, J., Zhang, J., Ibrahim, P.N., Cho, H. et al. (2010) Clinical efficacy of a RAF inhibitor needs broad target blockade in BRAF-mutant melanoma. Nature 467, 596-599.

74) Weinstein, I.B. (2002) Cancer. Addiction to oncogenes-the Achilles heal of cancer. Science 297, 6364.

75) Nazarian, R., Shi, H., Wang, Q., Kong, X., Koya, R.C., Lee, H. et al. (2010) Melanomas acquire resistance to B-RAF(V600E) inhibition by RTK or N-RAS upregulation. Nature 468, 973-977.

76) King, A.J., Arnone, M.R., Bleam, M.R., Moss, K.G., Yang, J., Fedorowicz, K.E. et al. (2013) Dabrafenib; preclinical characterization, increased efficacy when combined with trametinib, while BRAF/ MEK tool combination reduced skin lesions. PLoS One 8, e67583.

77) Yaeger, R., Yao, Z., Hyman, D.M., Hechtman, J.F., Vakiani, E., Zhao, H. et al. (2017) Mechanisms of acquired resistance to BRAF V600E inhibition in colon cancers converge on RAF dimerization and are sensitive to its inhibition. Cancer Res. 77, 6513-6523.

78) Chen, S.H., Zhang, Y., Van Horn, R.D., Yin, T., Buchanan, S., Yadav, V. et al. (2016) Oncogenic BRAF deletions that function as homodimers and are sensitive to inhibition by RAF dimer inhibitor LY3009120. Cancer Discov. 6, 300-315.

79) Cotto-Rios, X.M., Agianian, B., Gitego, N., Zacharioudakis, E., Giricz, O., Wu, Y. et al. (2020) Inhibitors of BRAF dimers using an allosteric site. Nat. Commun. 11, 4370.

80) Luebker, S.A. and Koepsell, S.A. (2019) Diverse mechanisms of BRAF inhibitor resistance in melanoma identified in clinical and preclinical studies. Front. Oncol. 9, 268.

81) Yen, I., Shanahan, F., Lee, J., Hong, Y.S., Shin, S.J., Moore, A.R. et al. (2021) ARAF mutations confer 
resistance to the RAF inhibitor belvarafenib in melanoma. Nature 594, 418-423.

82) Hatzivassiliou, G., Song, K., Yen, I., Brandhuber, B.J., Anderson, D.J., Alvarado, R. et al. (2010) RAF inhibitors prime wild-type RAF to activate the MAPK pathway and enhance growth. Nature 464, 431-435.

(Received Oct. 26, 2021; accepted Dec. 14, 2021)

\section{Profile}

Yoshiro Niitsu was born in Obihiro city, Hokkaido, Japan in 1943. After graduation from Sapporo Medical College in 1967 and finishing a 1-year internship at the Medical Hospital, he selected internal medicine as his major course. During 4 years on the graduate course at Sapporo Medical College, he spent 6 months as a research associate in the Department of Nutrition, MIT, Boston to collaborate on the posttranscriptional regulation of an iron binding protein, ferritin, and spent another year as an exchange graduate student at the Department of Biophysics, Osaka University to study the physicochemical properties of ferritin and immunoglobulin. Soon after receiving this $\mathrm{PhD}$ in 1972, he joined the research group of Dr. Irving Listowsky at the Department of Biochemistry, Albert Einstein College of Medicine, NY as a postdoctoral fellow and

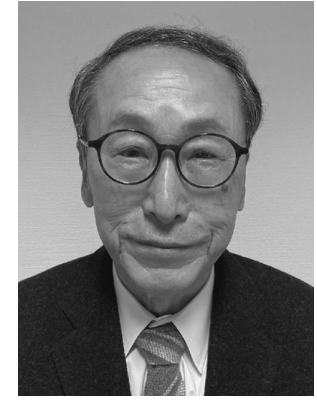
continued his studies on the physicochemical property of ferritin and ligandin. He started his clinical research career upon returning to Sapporo Medical College where he later became professor of the Department of Internal Medicine (1988-2008). As with most professorial members of the Department of Internal Medicine at that time, he had to cover a wide range of specialties, which included hematology, clinical oncology, and gastroenterology. He was appointed twice as vice director of Sapporo Medical College Hospital. He is currently an adviser of Oncology Section, Center of Advanced Medicine, Shonan Kamakura General Hospital. He received the Hokkaido Governor's award in 2004, Hokkaido Science Technology award in 2004, Princess Takamatsu Cancer Research Fund Prizes of the year Heisei 16 (2004) and the outstanding achievement award of the International Symposium on Molecular Medicine in 2005. He was selected as Nice Step Scientist by the National Institute of Science and Technology Policy in 2008. He was conferred with the honor of The Order of the Sacred Treasure in 2021. 